\title{
Criminologie
}

\section{Justice et infra-justice en Nouvelle-France. Les voies de fait à Montréal entre 1700 et 1760}

\section{Jean-François Leclerc}

Volume 18, numéro 1, 1985

L'histoire du contrôle social

URI : https://id.erudit.org/iderudit/017205ar

DOI : https://doi.org/10.7202/017205ar

Aller au sommaire du numéro

\section{Éditeur(s)}

Les Presses de l'Université de Montréal

ISSN

0316-0041 (imprimé)

1492-1367 (numérique)

Découvrir la revue

Citer cet article

Leclerc, J.-F. (1985). Justice et infra-justice en Nouvelle-France. Les voies de fait à Montréal entre 1700 et 1760. Criminologie, 18(1), 25-39.

https://doi.org/10.7202/017205ar

\section{Résumé de l'article}

It has become obvious in the past several years that the judicial statistics have certain limitations as far as reconstructing the evolution of crime under the Ancien Régime is concerned. The administrative inadequacies of the institutional justice of that era, its insufficient means of intervention, invite caution, but the existence of infra-judicial mechanisms for dealing with crime lead to the conclusion that only a fraction of the crimes ever came before the courts. Our research on trials for assault and battery heard in the jurisdiction of Montreal between 1700 and 1760 revealed several cases establishing the existence in New France, and confirming the observations of Alfred Somanfor France, of practices according to which the institution of proceedings by certain victims was often in order to start a process of negotiation with the aggressor or force him to come to an agreement out of court, which usually put an end to the procedures. Since there is rarely any explicit mention of agreements reached, even in the notarial archives, we arrived at the possibility that the dropping of legal proceedings indicated an infra-judicial settlement of the case. This led us to analyse the decrease in the number of trials for assault and battery in Montreal after 1730. It was not because of the usual explanation, that it was a sign of a "decrease in crime", but rather due to a strengthening of the infra-judicial system, particularly in the rural areas, where there was less recourse to the authority of the royal court to settle minor infractions.
Tous droits réservés @ Les Presses de l'Université de Montréal, 1985
Ce document est protégé par la loi sur le droit d'auteur. L’utilisation des services d'Érudit (y compris la reproduction) est assujettie à sa politique d'utilisation que vous pouvez consulter en ligne.

https://apropos.erudit.org/fr/usagers/politique-dutilisation/ 


\section{JUSTICE ET INFRA-JUSTICE EN NOUVELLE-FRANCE LES VOIES DE FAIT À MONTRÉAL ENTRE 1700 ET 1760.* Jean-François Leclerc*}

It has become obvious in the past several years that the judicial statistics have certain limitations as far as reconstructing the evolution of crime under the Ancien Regime is concerned. The administrative inadequacies of the institutional justice of that era, its insufficient means of intervention, invite caution, but the existence of infra-judicial mechanisms for dealing with crime lead to the conclusion that only a fraction of the crimes ever came before the courts. Our research on trials for assault and battery heard in the jurisdiction of Montreal between 1700 and 1760 revealed several cases establishing the existence in New France, and confirming the observations of Alfred Soman for France, of practices according to which the institution of proceedings by certain victims was often in order to start a process of negotiation with the aggressor or force him to come to an agreement out of court, which usually put an end to the procedures. Since there is rarely any explicit mention of agreements reached, even in the notarial archives, we arrived at the possibility that the dropping of legal proceedings indicated an infra-judicial settlement of the case. This led us to analyse the decrease in the number of trials for assault and battery in Montreal after 1730. It was not because of the usual explanation, that it was a sign of a "decrease in crime», but rather due to a strengthening of the infra-judicial system, particularly in the rural areas, where there was less recourse to the authority of the royal court to settle minor infractions.

\section{INTRODUCTION}

On perçoit mieux depuis quelques années avec quelle prudence particulière le chercheur en histoire de la justice et de la criminalité se doit d'aborder les archives judiciaires de l'Ancien Régime pour assurer la justesse de ses interprétations. Les registres criminels et les greffes judiciaires sont loin de constituer un relevé exhaustif reflétant fidèlement la nature et l'évolution de la criminalité dans une société donnée. Les carences administratives de la justice institutionnelle de l'époque ainsi que ses faibles moyens de surveillance et d'intervention l'expliquent en

* Cet article se fonde sur un aspect des recherches portant sur les délits de voie de fait dénoncés au tribunal royal de Montréal, entre 1700 et 1760 , recherches réalisées dans le cadre d'un mémoire de maûtrise au Département d'histoire de l'Université de Montréal.

** M. Jean-François Leclerc, assistant de recherche, Departement d'histoire, Université de Montréal. 
partie. Mais c'est surtout l'existence de mécanismes infra-judiciaires de règlement des délits qui permet de penser qu'une fraction seulement de la criminalité se retrouve devant les tribunaux réguliers.

Si les recherches historiques sur la France de l'Ancien Régime n'ont pas encore permis de mesurer exactement l'importance réelle de l'infrajustice dans le système de contrôle social français des XVIIe et XVIIIe siècles, elles en ont indubitablement démontré l'existence ${ }^{1}$. On ne saurait en dire autant des études portant sur le système judiciaire implanté en Nouvelle-France sur le modèle métropolitain. Les travaux d'André Lachance sur la justice criminelle et de John A. Dickinson sur la justice civile s'intéressent surtout à décrire l'organisation, le fonctionnement et les activités du système judiciaire ainsi que les litiges et les délits enregistrés par les tribunaux de la colonie sans prêter une attention particulière aux mécanismes infra-judiciaires de règlement $t^{2}$. Nos recherches sur les délits de voie de fait ${ }^{3}$ portés à la connaissance du tribunal royal de Montréal entre 1700 et 1760 nous ont permis d'en retrouver plusieurs pratiques semblables à celles observées dans la métropole à la même époque. Il n'en fallait pas plus pour soulever le problème des rapports entre la justice et l'infra-justice dans cette société coloniale française.

\section{UN RECOURS CONTRAIGNANT}

C'est en 1693 que le roi établit à Montréal un tribunal royal de première instance, dont la juridiction s'étend à tout le territoire du gouvernement de Montréal, comprenant les zones habitées des deux rives du Saint-Laurent à l'ouest de Sorel, et celles de la Rivière Richelieu ${ }^{4}$. Les

1. Parmi les nombreux travaux de Nicole Castan, mentionnons Justice et répressions en Languedoc a l'époque des Lumières, Paris, Flammarion, 1980. Voir aussi de Nicole et Yves Castan, «Une économie de justice à l'âge moderne : composition et dissension", dans la revue Histoire, économie et société, $1^{\mathrm{re}}$ année, $1^{\mathrm{er}}$ trimestre 1982, pp. 361-367. L'article d'A. Soman dans la revue Criminal Justice History, vol. I, 1980. "Deviance and Criminal Justice in Western Europe, 1300-1800: An Essay in Structure» a très bien dégagé la problématique de la recherche sur la question.

2. André Lachance, la Justice criminelle du roi au Canada au xvmre siècle. Tribunaux et officiers, Les Cahiers d'histoire de l'Université Laval, Québec, Les Presses de l'Université Laval, 1978. Crimes et criminels en Nouvelle-France, Montréal, Les Éditions du Boréal Express, 1984. John A. Dickinson, Justice et justiciables. La procédure civile à la Prévoté de Québec, 1667-1759, Les Cahiers d'histoire de l'Université Laval, Québec, Les Pesses de I'Université Laval, 1982.

3. Sous ce vocable sont regroupés toutes les atteintes physiques non mortelles causant ou non des blessures.

4. Au moment de la création de ce tribunal, il y a déjà plus de 40 ans que les seigneurs de l'île de Montréal (d'abord la Société Notre-Dame, puis les Sulpiciens) exercent leurs droits de justice dans les limites de leur seigneurie. La juridiction de ce tribunal sei- 
appels de ces décisions relèvent du Conseil supérieur, qui siège à Québec. Alors qu'en France, les pouvoirs judiciaires s'exercent à travers un réseau très complexe de tribunaux aux juridictions souvent concurrentes ${ }^{5}$, les structures judiciaires de la Nouvelle-France donnent à la justice royale un rôle prépondérant. La mise en place du système seigneurial au $X V \Pi^{e}$ siècle avait déjà donné lieu à la création de tribunaux locaux dotés de compétences pénales mais il semble qu'au XVIII siècle, la plupart d'entre eux laissent aux tribunaux royaux le soin de juger les crimes et délits commis sur leur territoire ${ }^{6}$.

Parmi toutes les plaintes entendues par les juges du tribunal royal montréalais ${ }^{7}$, celles dénonçant les voies de fait sont certainement les plus communes. Il est toujours étonnant pour nous de constater la spontanéité avec laquelle en ce «siècle des Lumières", on règle publiquement ses querelles à coups de canne ou de poing, sans susciter pour autant un émoi particulier chez les témoins de l'incident. Cette violence, sans être banale, ponctue les rapports humains même dans des milieux aisés

gneurial avait été étendue en 1683 à l 'ensemble du gouvernement. Divers facteurs, comme l'importance accrue de la ville sur le plan militaire et économique avec les difficultés que cela comporte, pousseront en 1693 les Sulpiciens à céder au roi la plupart de leurs droits de justice, ne conservant que leur juridiction sur certains litiges de nature civile.

5. Pierre Goubert parle au sujet de la justice de l'Ancien Régime de véritable «imbroglio administratif». Voir le chapitre consacré aux tribunaux dans son ouvrage l'Ancien Régime, t. 2, les Pouvoirs, Paris, Armand Colin, 1973, pp. 94-111.

6. Notons que l'intendant, comme premier juge de la colonie, possède aussi d'importants pouvoirs judiciaires qui lui permettent d'évoquer devant lui et juger de toute affaire tant civile que criminelle, et arbitrer toute contestation sommaire à la demande des parties ou conférer ce pouvoir à ses subdélégués. Le gouverneur général et ses représentants locaux jouissent aussi d'une certaine autorité sur le plan judiciaire. Il est difficile d'évaluer la fréquence de leurs interventions, mais la gratuité de leurs jugements les rend certainement attrayantes. L'intendant Claude-Thomas Dupuy, en poste de 1725 à 1728 affirme qu'on vient joumellement lui demander justice pour des affaires légères, surtout de nature civile, mais des voies de fait s'y glissent à l'occasion. Les interventions judiciaires de ces gouvemants dans les cas de litiges et de délits relevant aussi des justices royale et seigneuriale n'ont fait l'objet d'aucune étude spécifique malgré leur impact probable sur les niveaux d'activité des tribunaux réguliers, dont témoignent les observations de J.A. Dickinson à propos de la Prévoté de Québec. Nicole Castan, a consacré dans son ouvrage sur la justice au Languedoc plusieurs pages à ces juges d'exception que sont les intendants, les gouverneurs et commandants dont on aurait tort d'ignorer l'influence. Toutefois, A. Lachance, qui a consulté les registres des intendants et gouverneurs, affirme n'y avoir trouvé aucun document se référant à des affaires criminelles. G. Lanctôt, l'Administration de la NouvelleFrance, Montréal, Éd. du Jour, 1971 ; J.-C. Dubé, Claude-Thomas Dupuy, intendant de la Nouvelle-France, Fides, 1969, p. 216 ; J. A. Dickinson, Justice et justiciables... , p. 104 ; N. Castan, Justice et répression en Languedoc..., pp. 195-211; A. Lachance, Crimes et criminels..., pp. 157-163.

7. Les dossiers judiciaires du tribunal royal de Montréal sous régime français sont conservés aux Archives nationales du Québec à Montréal. La mention DJ dans les prochaines notes y fera référence. 
qui de nos jours tolèreraient mal un tel manquement aux règles de la civilité. Bien que les voies de fait représentent le délit le plus fréquemment jugé dans les tribunaux de l'époque ${ }^{8}$, tous les Montréalais à la main leste ne se retrouvent pas automatiquement sur le banc des accusés. La faiblesse des effectifs policiers, commune aux appareils judiciaires d'Ancien Régime, ne permet pas d'appréhender sur le fait tous les contrevenants ${ }^{9}$. De plus, la voie judiciaire présente certains obstacles qui limitent certainement, dans le cas des voies de fait, le recours spontané à l'autorité d'un juge royal.

En dépit des intentions royales, la justice institutionnelle demeure en effet relativement onéreuse pour la plupart des justiciables. La Couronne laisse en général l'initiative et le coût des poursuites criminelles pour des délits (comme les voies de fait) touchant les particuliers à la charge des personnes lésées, n'intervenant à ses frais que dans les cas les plus graves ou lorsque l'ordre public est menacé. Le plaignant doit normalement assumer les frais judiciaires jusqu'à ce qu'un jugement reconnaisse la culpabilité de l'accusé et le condamne à les rembourser ${ }^{10}$. À l'influence dissuasive des coûts renforcée par le facteur de l'éloignement et l'obligation de suspendre ses activités professionnelles ou ses occupations journalières s'ajoute l'aspect rebutant des méthodes intimidantes de la justice criminelle que rappellent à l'occasion les châtiments spectaculaires exécutés sur la place publique.

\section{LA VOIE DE L'ACCOMPAGNEMENT}

Les justiciables qui hésitent pour ces raisons à faire appel à l'autorité du tribunal ne sont pas pour autant dépourvus de moyens d'obtenir justice. Les mécanismes d'arbitrage fréquemment utilisés pour trancher des litiges de nature civile se prêtent au règlement de certains délits même

8. A. Lachance, Crimes et criminels..., P. 26.

9. À Montréal, il existe une maréchaussée formée seulement d'un prévôt et de trois archers. La garde militaire, et les officiers de milice urbains et nuraux veillent aussi probablement au maintien de l'ordre, mais à en juger par les récits judiciaires de voies de fait, leurs interventions semblent assez rares et surtout inconstantes. L'état actuel des connaissances sur leur rôle effectif dans le système de contrôle social de la colonie ne permet pas pour le moment d'en dire plus.

10. Les frais de procès sont connus dans une minorité de cas, habituellement lorsqu'une sentence vient en préciser le montant. Pour la plupart d'entre eux $(70 \%)$ les dépens oscillent entre 20 et 60 livres, mais une part appréciable (15\%) atteint plus de 100 livres. Or on estime le salaire moyen d'un artisan de l'époque, à 600 livres au maximum par année dans les meilleures conditions. Voir Cameron Nish, les Bourgeois-gentilhommes de la Nouvelle-France 1729-1748, Fides, 1968, p. 41. 
graves $^{11}$, et plus encore des voies de fait aux conséquences relativement bénignes. Les demandes des victimes, lorsqu'elles sont clairement exprimées dans les plaintes déposées au greffe du tribunal, consistent généralement dans la réparation des torts moraux et physiques, le principe fondamental étant, comme le note Nicole Castan «d'effacer l'agression et toutes ses séquelles» ${ }^{12}$. Les modalités d'une réparation d'honneur, le paiement de soins médicaux, la récupération des pertes d'argent causées par la maladie et l'incapacité temporaire à gagner sa vie sont des choses tout à fait négociables avec l'aide de quelque notable ou ami, comme le serait l'évaluation de dommages matériels, par exemple le ravage d'un potager par quelques vaches ou le bris d'une clôture. C'est ainsi qu'en février 1753, deux jeunes gens de Sorel s'entendent par l'entremise de leurs parents, en présence d'officiers de milice de l'endroit, avec un marchand victime de leurs mauvais traitements pour payer 25 livres à l'église paroissiale (une forme de réparation morale en substitut à l'amende judiciaire), les médicaments du chirurgien et pour faire ses travaux jusqu'à son entière guérison ${ }^{13}$.

Si de telles ententes à l'amiable paraissent souhaitables, elles ne sont pas nécessairement désirées par l'une ou l'autre des parties malgré les efforts concertés de leur entourage pour les en convaincre. En 1732, l'huissier Nicolas Marchand, qui s'apprêtait à saisir une vache, est assailli par Marcel Corbin, un laboureur au service d'une communauté religieuse. L'huissier ayant fait venir un chirurgien, les religieuses envoient demander la femme de la victime "pour le prier de surseoir la plainte et leur donner un peu de temps", ce qu'il accepte. Sans tarder, les religieuses mandent le colonel de la milice du gouvernement de Montréal, le sieur Étienne Volan de Radisson d'accommoder l'affaire. Mais l'obstination du huissier est telle que les offres de dédommagement de la part des

11. Même pour des affaires de meurtre, quoique dans ces cas graves, une permission de la magistrature ou une lettre de rémission royale soit nécessaire. A. Soman, "L'infrajustice à Paris d'après les archives notariales», Histoire, économie et société, $1^{\text {re }}$ année, $1^{\text {er }}$ trimestre 1982 , p. 374. Étonnement, les rébellions à justice (obstruction au travail des huissiers) semblent aussi se prêter à de tels règlements privés, comme le révèlent plusieurs dossiers du tribunal royal montréalais.

12. N. Castan, Justice et répression en Languedoc..., p. 50. Notons qu'il arrive, assez rarement il est vrai, que des plaignants requièrent ouvertement l'arrestation de leur agresseur et la répression du délit. Mais la jurisprudence en matière de voies de fait prévoit en général des compensations matérielles assorties d'une amende.

13. DJ, 7 février 1753 . 
religieuses à l'occasion d'interventions répétées du colonel et d'une noble dame ne parviendront pas à le faire céder ${ }^{14}$.

Les difficultés rencontrées lors de négociations, tout comme le refus d'un agresseur de reconnaître sa responsabilité par un accord ou l'incapacité de réparer monétairement ses torts, peuvent déterminer une victime à porter ses réclamations devant le juge royal de la juridiction. Un exposé habilement tourné selon les règles de la rhétorique judiciaire peut facilement convaincre le juge d'autoriser des poursuites ${ }^{15}$. La victime est dépeinte innocente, faible et sans défenses, l'agresseur, d'une fureur animale et nourrissant les plus noirs desseins, un tableau dramatique auquel la justice ne reste pas insensible quoique l'enquête réduise souvent l'affaire à des proportions plus banales.

L'issue pénale d'une affaire soumise à l'attention du tribunal, malgré ce qu'on pourrait croire, n'est pas inéluctable. Alfred Soman a bien fait ressortir le rôle éminemment stratégique d'une telle démarche ${ }^{16}$. Le plaignant, qui pour diverses raisons, ne craint pas de saisir à ses frais la justice criminelle d'un délit de voie de fait, dispose en effet de moyens de pression considérables. La menace d'une arrestation causant la "confusion d'être amené comme un criminel», selon l'expression d'un accusé, a tôt fait de convertir les plus récalcitrants aux vertus de la bonne entente, compte tenu également de l'éventualité d'avoir à supporter des frais judiciaires parfois plus considérables que les dommages et intérêts normalement imposés pour de tels délits ${ }^{17}$. Des négociations engagées ou reprises dans de telles circonstances prennent dès lors un caractère impérieux auquel il est difficile de se soustraire. L'amorce d'un combat sur le terrain judiciaire, où le plaignant assuré de sa preuve occupe une posi-

14. DJ, 16 mai 1732. Marcel Corbin déclare de son côté «qu'il ne donnerait un cheveu de sa perruque" pour l'huissier. Les efforts déployés par les religieuses pour apaiser l'affaire, malgré cette obstination, viendraient selon l'huissier, de leur désir de récupérer une somme d'argent que leur doit leur laboureur et qu'elles perdraient si jamais une accusation le forçait à s'enfuir.

15. Le nombre des plaignants faisant appel pour les représenter à des personnes conaisssant les rouages judiciaires plutôt qu'à des parents ou des amis, tend à s'accroître au cours du XVIme siècle. Mais en général, la plupart se contentent vraisemblablement d'exposer eux-mêmes les circonstances du délit au greffier du tribunal.

16. A. Soman, "Déviance and Criminal Justice...», p. 10.

17. Les frais judiciaires dans le cas des procès pour voie de fait à Montréal égalent ou dépassent fréquemment les dédommagements alloués aux victimes. À moins d'être déchargé des accusations, même lorsque la culpabilité est incertaine, un accusé est au minimum condamné à payer les dépens. 
tion privilégiée ${ }^{18}$, permet de sonder l'adversaire et de préparer la voie à l'accommodement le plus avantageux.

Lorsque les réclamations n'obligent pas l'accusé à des concessions trop onéreuses ou humiliantes, les pressions judiciaires suffisent à renouer les liens. En septembre 1723, le cordonnier Bernard Dumouchel s'exclame au passage du maître tonnelier Jacques Hery Duplanty «Bougre tu as l'effronterie de passer devant moi sans ôter ton chapeau», puis s'empresse de réagir à ce manque d'égards en le frappant de plusieurs coups dont un «dans les parties génitales». Mal en point, le tonnelier dépose le jour même une plainte au tribunal royal, demande la sauvegarde du roi et de la justice et obtient une enquête qui confirme en la nuançant sa version de l'incident. Un décret «d'ajournement personnel» ${ }^{19}$ est lancé contre le cordonnier, mais celui-ci ne comparaît devant le juge royal que douze jours plus tard. Il se dit alors :

très surpris que ledit Duplanty poursuive cette affaire puisqu'ils se sont accommodés ensemble et qu'il lui a demandé excuse et pardon à genoux en présence des nommés Fleur d'épée Étienne Petit et le Sr De Bailleul chez La giroflée le priant de vouloir oublier cette affaire et que ledit Duplanty lui promit et restèrent presque toute la journée chez ledit La giroflée en présence des susnommés à boire et à manger et qu'il a payé la dépense qui se fit pour lors jusqu'à un verre qui fut cassé.

L'affaire en serait restée là, si à la suite de ces agapes bien arrosées, le tonnelier Duplanty ne s'était mis en tête, selon l'accusé, de récupérer aussi les frais judiciaires encourus jusque-là «dont pour lors de leur accommodement ils n'en avaient point parlé». La sentence lui donna satisfaction. Le cordonnier fut condamné aux dépens (frais judiciaires), le

18. *L'assurance des "plaintifs" vient de leur assimilation de la pratique et de leur certitude qu'une voie de fait bien établie contraint le juge à imposer une peine dont la légèreté est indifférente si la note de frais est très lourde.» $\mathrm{N}$. Castan, «Une économie de justice...", p. 366.

19. Le décret est une ordonnance rendue par le juge sur la foi de l'information (l'enquête criminelle) et des conclusions du procureur du roi, par lequel l'accusé est appelé à comparaître pour répondre à l'accusation intentée contre lui. Il existe trois sortes de décrets : le décret de prise de corps, qui équivaut à une arrestation; le décret d'ajournement personnel, ordonnant la comparution de l'accusé en personne; et enfin le décret d'assigné pour être ouï, appelant l'accusé à être entendu sur les faits révélés par l'information. Le degré de gravité des charges pesant contre les accusés détermine le juge d'émettre selon le cas l'un ou l'autre de ces décrets. Pour toutes les questions touchant la procédure criminelle et les peines dans le droit français d'Ancien Régime, voir A. Laingui, A. Lebigre, Histoire du droit pénal, I-II, Paris, Cujas, 1979. 
juge ne faisant en somme qu'imposer d'office des frais dont le paiement était normalement prévu lors de la conclusion d'un accord ${ }^{20}$.

S'il est parfois possible de se réconcilier en trinquant chez le cabaretier, il en va tout autrement lorsque survient un incident entre querelleurs aux rancunes anciennes et tenaces. Le recours à la procédure criminelle devient dans certains cas l'occasion de réaliser de purs desseins de vengeance qui entravent le processus de réconciliation. En juin 1699, l'incursion des boufs de Charles Brazeau fils dans les blés de son voisin le boulanger François Blot, provoque une rixe dont le fermier et sa femme enceinte sortent passablement meurtris ${ }^{21}$. Rencontrant le Sieur Jacques Testard de Montigny, officier militaire bien connu ${ }^{22}$ dont Charles Brazeau est le fermier, François Blot se félicite de la conduite de ses enfants, tout comme sa femme qui aurait souhaité "qu'il lui eussent cassé le col». Il va même jusqu'à brandir la menace d'un coup de fusil qui casserait les jambes de son adversaire. L'affaire ne tarde pas à rebondir en justice puisque le même jour, Charles Brazeau dépose une plainte judiciaire mettant principalement en cause un des fils de son voisin et leur vacher. Malgré ses bravades, le boulanger est vivement impressionné par les suites de l'affaire : le fermier Brazeau appelle au chevet de sa femme un chirurgien qui confirme l'état critique de cette dernière en danger d'avortement et même de mort. Le mari fait même venir le prêtre pour lui administrer les derniers sacrements, signe spectaculaire dont Brazeau profite pour laisser entendre à son voisin que ses fils seront pendus pour ce crime. Deux jours après la tenue de l'enquête, Brazeau envoie néanmoins chercher son beau-père, un huissier, et ils se rendent ensemble chez le boulanger pour lui proposer de terminer l'affaire. Les parties s'assemblent chez le Sieur de Montigny, en présence de témoins. Sous la menace d'une prétendue sentence dont son beau-père serait porteur, le plaignant réussit à faire accepter au boulanger de payer la somme considérable de 725 livres, pour couvrir les soins du chinurgien, les dommages et les frais judiciaires. La transaction est passée devant notaire pour "conserver entre eux leur ancienne et bonne amitié", et prévoit l'abandon des poursuites judiciaires quel que soit le sort futur de la malade. Les accusés, le boulanger et ses fils, promettent de ne pas récidiver et reconnaissent les plaignants pour gens de bien. Le Sieur de Mon-

20. DJ, 16 septembre 1723.

21. Une autre querelle reliée à une affaire semblable venait d'opposer les deux familles.

22. Voir sa biographie dans le Dictionnaire biographique du Canada, vol. II, pp. 653-655. 
tigny lui-même avance au boulanger les 725 livres que ce dernier s'engage, par une obligation, à rembourser à la volonté du créancier, ses biens étant à cet effet hypothéqués...

Le renouement forcé et expéditif de leur «ancienne et bonne amitié» ne fut pas de longue durée, car, dès le lendemain de la signature de l'accord, la femme prétendument mourante du fermier paraissait si bien remise de son choc qu'elle vaquait à ses occupations ordinaires comme s'il ne s'était rien passé. Trompés, menacés de saisie par leur créancier de Montigny, les accusés tentèrent de contester l'entente mais n'obtiendront que la reprise du procès dont ils sortiront néanmoins perdants ${ }^{23}$.

Les avantages évidents qui découlent de la position de plaignant n'éliminent cependant pas complètement les risques de l'aventure judiciaire. Si la plainte est mal étayée, ou trop peu incriminante, le plaignant risque de se voir condamné aux dépens et même à des dommages et intérêts ${ }^{24}$. Les accusés ont alors beau jeu de lui faire payer par une amère revanche cette atteinte à leur réputation ${ }^{25}$.

La méconnaissance des atouts défensifs d'un adversaire peut à l'occasion réserver de mauvaises surprises. En 1725, un jeune homme de la Rivière-des-Prairies met en branle la justice royale après avoir été battu par un rival au sujet d'une jeune fille que tous deux fréquentaient. Cette poursuite judiciaire impressionne si bien le père de l'accusé, que celui-ci, effrayé par l'arrivée d'un huissier accompagné d'un sergent et de deux soldats venus arrêter son fils, absent, accepte de transiger un accord stipulant le paiement de 250 livres et des frais de justice, et forçant son fils à demander pardon à son rival en présence du curé et des quatre plus notables de la paroisse. À son retour, l'accusé conteste l'accord, affirmant qu'on avait trompé son père, un homme qui *n'entend point les affaires", et soulignant le "tort notable que cela ferait à son honneur et à sa réputation». Ne lésinant pas sur les moyens, il paie les

\section{DJ, 17 juin 1699.}

24. Si la chose est théoriquement possible, peu de plaignants ont connu ce sort en ce qui concerne les voies de fait. Le cas le plus notable se produit en 1707, alors que l'aide-major de Montréal est condamné à payer des frais de 198 livres pour le procès intenté par lui contre un voyageur et un couturier de l'endroit. Voir DJ, 9 juillet 1707.

25 . En 1731 , le procès intenté par un cultivateur contre des jeunes voyageurs est converti en procès ordinaire, ce qui laisse prévoir que les accusés n'auront à essuyer qu'une peine pécuniaire. Près d'un mois plus tard, le cultivateur dans une requête au juge, se plaint que son procès est en suspens parce qu'il n'a qu'un témoin, et que les accusés se croyant déchargés des accusations se sont mis à l'insulter et composer des chansons «infâmes» à son sujet. DJ, 17 octobre 1731 . 
services d'un huissier qui fait le tour de l'île Jésus et de la côte Rivièredes-Prairies, recueillant une soixantaine d'appuis des personnes les plus importanes de ces lieux, chacune certifiant à sa façon que l'accusé est «honnête homme». Si cette riposte ne le libère pas des poursuites ${ }^{26}$, elle permettra à l'accusé d'obtenir une réparation d'honneur, avec dommages et dépens, une victoire d'autant plus gratifiante que la publicité donnée à l'incident par la tournée du huissier l'aura rendue retentissante ${ }^{27}$ !

\section{INFRA-JUSTICE ET DÉCROISSANCE DU CRIME}

Les procédures ne nous livrent pas toujours de façon aussi étoffée le récit du processus parfois complexe menant à un règlement hors cour. Quand rien n'est venu interrompre une négociation, ou remettre en cause un accommodement, le dossier judiciaire se clôt laconiquement sur la mention d'un désistement ou la brève description des termes d'une entente. Pourtant, dans un cas comme dans l'autre, elles démontrent sans l'ombre d'un doute l'existence d'une infra-justice en Nouvelle-France au XVIII siècle.

Cette constatation ne facilite pas l'analyse de l'évolution du système judiciaire et de la criminalité dans l'Ancien Régime. Comment peut-on en effet interpréter correctement les statistiques judiciaires et leurs fluctuations si une partie indéterminée des crimes et des délits échappent à notre observation? Sommes-nous condamnés aux extrapolations hasardeuses dont parle Alfred Soman à propos de certaines études quantitatives ${ }^{28}$ ?

Au cours du XVnie siècle, il est frappant de constater une nette diminution de la fréquence des délits de violence portés devant les tribunaux de plusieurs juridictions tant en France qu'au Canada ${ }^{29}$. Ainsi, à Mont-

26. Il sera libéré sous caution à la suite d'une sentence de "plus ample informé» quj laisse planer la menace d'une reprise de la poursuite si des faits nouveaux et incriminants viennent étayer l'affaire.

27. DJ, 17 septembre 1719.

28. A. Soman, «L'infra-justice à Paris.., p. 369.

29. En plus de l'ouvrage déjà cité d'A. Lachance sur la criminalité en NouvelleFrance, nous nous refétons pour la France aux articles suivants : M.M. Champin, «La criminalité dans le baillage d'Alençon de 1715 à 1745», Annales de Normandie, t. 22, 1972, pp. 47-84. A. Farouk, «Rupture et mutation de la justice royale. Le bailliage royal

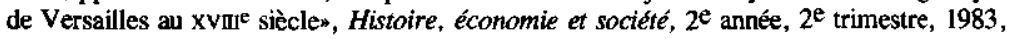
pp. 303-335. A. Margot, «La criminalité dans le bailliage de Maniers 1695-1750", Annales de Normandie, t. 22, 1972. N. W. Mogensen, aCrimes and Punishments in EighteenthCentury France. The Example of the pays d'Auge», Histoire sociale, vol. 10, $\mathrm{n}^{\circ} 20$, novembre 1977, pp. 337-353. 
réal, la moyenne annuelle des causes de voie de fait entendues devant le tribunal royal passe de 6 à 1 entre 1720 et 1760 . La rapidité d'un tel phénomène et sa coïncidence dans des endroits aussi éloignés que Mamers en Normandie, Versailles, et Montréal, posent plus d'énigmes qu'elles n'en résolvent. On ne peut sans hésitation souscrire comme certains l'ont fait à l'hypothèse d'une «admirable» régression de la violence ${ }^{30}$, conséquence d'un contrôle tardif de "l'hyperirritabilité" " ${ }^{31}$ traditionnelle des contemporains. À notre avis, des facteurs liés à l'évolution du système judiciaire, au rapport entre la justice et l'infra-justice, permettent peut-être mieux d'expliquer le déclin statistique des violences au XVIII e siècle, du moins en ce qui concerne les voies de fait à Montréal.

À prime abord, la démonstration de cette hypothèse se heurte à un problème délicat : la disparition d'une partie indéterminée des ententes infra-judiciaires, celles qui furent conclues verbalement (cf. l'affaire Duplanty-Dumouchel), ou encore devant des notables dont les documents n'ont pas été conservés ${ }^{32}$. L'obstacle est de taille lorsqu'on cherche à évaluer l'importance des mécanismes de règlement dans le contrôle des délits à l'époque, mais non infranchissable. En effet, plusieurs indices nous permettent de penser qu'en raison de son rôle dans la mise en marche du processus de négociation, la justice officielle garde certains signes mesurables des pratiques infra-judiciaires. À en juger par les accords conservés dans quelques greffes notariés de la région montréalaise, rares semblent les ententes conclues devant notaire sans aucun prélude judiciaire $^{33}$. Alfred Soman a noté le même usage à Paris en explorant

30. Selon l'expression de l'historien E. LeRoy Ladurie, «La décroissance du crime... au XVIII ${ }^{e}$ siècle : bilan d'historiens', Contrepoint, $\mathrm{n}^{\circ} 9,1973$.

31. N.W. Mogensen, op. cit., p. 340. Cette hypothèse n'a pas perdu sa popularité. Dans un récent article sur le bailliage de Versailles, $\mathrm{A}$. Farouk décrivait le déclin des délits dans cette juridiction comme le passage đ'une société modérément violente à une société calme où la délinquance n'apparaît que par intermittence». A. Farouk, op. cit., p. 313.

32. Les deux jeunes gens de Rivière-des-Prairies dont nous avons cité plus haut les démêlés, avaient conclu quelque temps plus tôt une entente, à la suite d'une affaire semblable, sous l'instigation du curé et du capitaine de milice de l'endroit. Ce dernier en aurait alors dressé un procès-verbal. DJ, 17 septembre 1719.

33. Nous avons consulté les inventaires des notaires suivants, publiés par les Archives de la Province de Québec dans la collection des Inventaires des Greffes des Notaires du Régime Français: J. H. Bouron (vol. XXIII),

J. David (vol. XII),

C.J. Porlier (vol. XV),

F. Coron (vol. XXII)

F. Lepailleur (vol. XXV)

J. C. Raimbault (vol. XXI)

Ces notaires ont exercé entre 1719 et 1760 dans les zones urbaines et rurales du gouvernement de Montréal. En tout, une cinquantaine d'accords, transactions, et conventions 
les archives notariales de la fin du XVII ${ }^{e}$ siècle $^{34}$. On peut donc présumer qu'une part appréciable des traces visibles de pratiques infrajudiciaires se retrouvent enregistrées indirectement dans les dossiers de la justice pénale montréalaise.

Bien peu de procès, il est vrai, une vingtaine ${ }^{35}$, font clairement référence à un désistement après entente, à un accord ou à des tentatives de négociation. Pourtant, comme nous l'avons constaté, l'abandon des poursuites avant le prononcé d'une sentence suit normalement la décision des parties de recourir à un accommodement plutôt qu'au jugement arbitraire d'un magistrat. Cet abandon, que révèle le caractère incomplet du dossier judiciaire, ne serait-il pas un indice assez sûr et mesurable du succès des démarches infra-judiciaires entreprises après l'amorce d'un procès?

Évidemment, le désistement du plaignant ou le renvoi des parties devant le tribunal civil pourrait aussi expliquer un arrêt prématuré des poursuites. Sans écarter cette possibilité, nous doutons que de telles décisions judiciaires aient été rendues sans être inscrites au dossier, alors qu'elles le sont en d'autres occasions. L'omission d'enregistrer les accords s'explique par contre aisément en raison du caractère privé de ces ententes, la justice étant tenue de ne plus poursuivre lorsque des transactions sont conclues dans le cas de délits mineurs ${ }^{36}$. Les huit (8) accords repérés dans quelques greffes notariés et liés à des poursuites judiciaires, ont été signés dans les jours suivant la production des dernières pièces de procédure sans qu'il n'en soit fait aucune mention dans les dossiers judiciaires correspondants. Notons que ces actes, comme les références à des accords ou des négociations dans les récits judiciaires, interviennent presque toujours au plus tard après les interrogatoi-

ont été repérés, mais la plupart se rapportent à des affaires civiles (ex. des partages de succession). Seuls huit (8) d'entre eux sont reliés à des poursuites judiciaires, tous pour voies de fait et se retrouvent sauf un cas dans le greffe de J.C. Raimbault, notaire et greffier au tribunal royal.

34. A. Soman, "L.'infra-justice à Paris...", p. 370.

35. 20 procès sur 239 soit environ $8 \%$. Ce taux rejoint celui calculé par Nicole Castan pour les procès criminels jugés en Languedoc à la fin du Xvmje siècle. N. Castan, Justice et répression..., p. 250.

36. Voir l'Ordonnance criminelle du mois d'août 1670 , pp. 371-423. Recueil général des Anciennes Lois françaises, t. XIX, par MM. Isambert, Taillandier et Decrusy, Paris, 1829. Selon un juriste de l'époque, l'usage français veut qu'il ne soit pas nécessaire qu'une transaction soit homologuée par le juge saisi du procès qu'elle termine. Guyot, G.-A., Répertoire universel et raisonné de jurispnudence civile, canonique et bénéficiale, Paris, Visse, 1784, XVI, p. 233. 
res, plus particulièrement à la suite de l'émission des décrets de comparution qui semblent convaincre les accusés du sérieux de l'affaire et de la détermination des plaignants. Or, la très grande majorité des procès abandonnés le sont aussi lors des premières étapes de la procédure ${ }^{37}$. À la lumière de ces observations, en apportant les nuances nécessaires, il nous semble assez juste de voir dans la baisse sensible des voies de fait et la diminution des taux d'abandon après 1730 , un indice permettant de saisir même imparfaitement l'évolution des pratiques infrajudiciaires à Montréal au XVIIIe siècle. De nos données à ce sujet, nous avons dégagé l'analyse suivante ${ }^{38}$.

\section{UNE HYPOTHĖSE}

Montréal au XVII' siècle est une société jeune, en pleine expansion démographique, la population triplant entre 1700 et $1760^{39}$. L'habitat dispersé, la faiblesse des structures d'encadrement dans un territoire trop vaste, ne favorisent pas comme le souligne Louise Dechêne ${ }^{40}$ la sociabilité normalement entretenue par les communautés de travail, de prière et de festivité, ou encore les relations de voisinage et de parenté propres aux sociétés plus anciennes. Quoique relativement faible mais non moins constante, l'immigration constitue aussi un facteur perturbant (10 à $15 \%$ de la population en 1715$)^{41}$. Les nouveaux venus sont temporairement

37. Aproximativement, voici la proportion des abandons suivant les pièces de procédure : plaintes $20 \%$

informations $20 \%$

décrets $40 \%$

interrogatoires $5 \%$

38. Nombre de procès pour voies de fait intentés dans la juridiction de Montréal 1700-1760 :

$\begin{array}{ccccccc}1700-1709 & 1710-1719 & 1720-1729 & 1730-1739 & 1740-1749 & 1750-1759 & \text { Total } \\ 32 & 60 & 64 & 48 & 22 & 13 & 239 \\ & 65 \% & & & 35 \% & & \end{array}$

Proportions de procès abandonnés ou se terminant par un accord 1700-1760.

$\begin{array}{cccccc}1700-1709 & 1710-1719 & 1720-1729 & 1730-1739 & 1740-1749 & 1750-1759 \\ 63 \% & 68 \% & 66 \% & 38 \% & 45 \% & 46 \%\end{array}$

39. D'après les Recensements du Canada, 1665 à 187I, IV, Ottawa, 1876. Entre 1698 et 1765, Montréal et ses faubourgs passent de 200 à 500 habitants; les campagnes de l'île de 500 à 4200 habitants; celles du reste du gouvernement de 1600 à 20000 habitants. (Ces données sont approximatives.)

40. L. Dechêne, Habitants et marchands de Montréal au XVIF siècle, Paris, Montréal, Plon, 1974, p. 434.

41. Ibid., p. 18. 
isolés en marge des natifs, et ne peuvent compter sur les fidélités et les appuis locaux pour régler leurs différends. Quant aux intermédiaires essentiels à toute négociation, l'absence de curés résidents et de capitaines de milice dans plusieurs côtes rurales, ainsi que la faiblesse des élites locales en voie de formation, les rend moins disponibles pour apaiser des conflits. Certains différends de nature civile, d'où originent fréquemment les voies de fait, peuvent aussi se retrouver à l'occasion d'une dispute devant le tribunal criminel à défaut d'avoir pu être réglé devant le tribunal civil (contrainte des coûts, incertitude des droits remis en cause par le litige) ou par un accommodement. Tous ces facteurs contribuent à porter plus spontanément les victimes de voies de fait, même légères, vers le recours judiciaire. La justice est donc souvent appelée à appuyer le processus de négociation peu soutenu par les moyens de pression locaux, d'où une croissance des délits de voie de fait jugés au tribunal royal pendant les deux premières décennies du XVחIe siècle.

Peu à peu, ces facteurs d'instabilité s'estompent. La société montréalaise mieux structurée, plus densément peuplée et resserrée par les liens familiaux élargis offre de meilleures conditions pour faire face localement aux délits de voie de fait. L'appui de la justice n'est plus aussi essentiel au déblocage des négociations. Les élites locales y veillant, il est probable que le renforcement des mécanismes infra-judiciaires pousse les juges à exercer une sélection plus sévère des poursuites, renvoyant les plus légères aux mécanismes locaux du règlement ${ }^{42}$; de plus, les justiciables eux-mêmes, mieux informés des exigences légales, saisissent moins spontanément le tribunal d'affaires légères, mal étayées et soumettent leurs litiges à l'arbitrage au lieu de les régler par la force ${ }^{43}$. Par conséquent, alors que s'amorce une diminution très marquée du nombre de poursuites intentées dans la juridiction de Montréal, la proportion des abandons passe de $60 \%$ qu'elle était avant 1730 , à $40 \%$ dans les décennies précédant la Conquête. Le déclin affecte essentiellement la part des procès non complétés, le nombre d'affaires menées jusqu'à

42. Un sondage très partiel effectué dans les registres d'audience de la juridiction de Montréal laisse croire que la décision de renvoyer les affaires de voie de fait devant la cour civile ou d'entendre les parties sans procéder à une enquête criminelle, intervient plus fréquemment dars les dernières décennies du Régime français. Cette pratique est d'ailleurs fortement recommandée par un juriste de l'époque, signe probable d'une certaine évolution de la sensibilité des magistrats en ce qui concerne les violences légères. Bornier, Philippe, Conférences des ordonnances de Louis XVI (...), Paris, 1755, p. 124.

43. Le déclin des activités dans les tribunaux au XVIII ${ }^{\mathrm{e}}$ siècle affecte aussi la justice civile, comme le montre l'étude de J.A Dickinson sur la Prévoté de Québec (op. cit.). L'auteur l'explique par un recours plus fréquent aux ententes à l'amiable, à la faveur de la formation de clans familiaux qui facilitent les relations sociales. 
une décision judiciaire demeurant à peu près le même que pendant la période précédente.

À la fin de l'Ancien Régime, selon Philippe Robert ${ }^{44}$, la justice régulière n'a qu'un but : ne pas avoir à s'exercer! L'effet dissuasif des coûts et de l'arbitraire des peines, qui tend à limiter le recours à son autorité, ne peut jouer pleinement que si d'autres moyens peu onéreux et efficaces permettent de règler simplement les délits. À Montréal les conditions idéales pour atteindre ce but seront probablement réunies à la fin du régime français, lorsque la stabilisation de la société coloniale favorisera l'enracinement de mécanismes de contrôle infra-judiciaires. Au point où la justice criminelle, à la veille de la Conquête, n'aura presque plus à intervenir pour juger des délits de voies de fait commis sur le territoire de sa juridiction ${ }^{45}$.

La complexité des problèmes soulevés par l'interprétation des statistiques judiciaires ne saurait être résolues sans un examen attentif des multiples rouages du système de contrôle social d'Ancien Régime. Mais dut-elle être approfondie, notre réflexion sur les délits de voies de fait à Montréal n'en pose pas moins la question de la place de l'infra-judiciaire dans la société de Nouvelle-France qui suscitera, espérons-le, d'autres recherches.

\section{Montréal.}

44. Séminaire tenu en octobre 1983 à l'École de criminologie de l'Université de

45. Nicole Castant a observé pour le Languedoc le phénomène inverse: l'effritement de ces moyens de régulation interne sera responsable de l'accroissement des recours judiciaires à la veille de la Révolution. N. Castan, Justice et répression... 